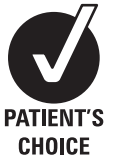

CHOICE

\title{
Social Cognition and Emotional Assessment differentiates frontotemporal dementia from depression
}

\author{
Maxime Bertoux, ${ }^{1,2,3,4}$ Marine Delavest, ${ }^{5,6}$ Leonardo Cruz de Souza, 1,2,3,4 \\ Aurélie Funkiewiez, ${ }^{3,4}$ Jean-Pierre Lépine, ${ }^{5,6}$ Philippe Fossati, ${ }^{7}$ Bruno Dubois, ${ }^{1,2,3,4}$ \\ Marie Sarazin ${ }^{1,2,3,4}$
}

${ }^{1}$ Université Pierre et Marie-Curie (UPMC) - Paris 6, Paris, France ${ }^{2}$ Institut du Cerveau et de la Moelle Epinière (ICM), INSERM UMRS 975, CNRS UMR 7225, Paris, France

${ }^{3}$ Institut de la Mémoire et de la Maladie d'Alzheimer (IM2A),

Hôpital Pitié-Salpêtrière, Paris, France

${ }^{4}$ Centre de Référence Démences Rares, Hôpital Pitié-Salpêtrière, Paris, France ${ }^{5}$ Service de Psychiatrie d'Adultes, Hôpital Fernand Widal - Lariboisière, Paris, France

${ }^{6}$ FondaMental Fondation Créteil, France

${ }^{7}$ Service de Psychiatrie d'Adultes, Hôpital

Pitié-Salpêtrière, Paris, France

Correspondence to Dr M Bertoux, Institut de la Mémoire et de la Maladie

d'Alzheimer - CHU

Pitié-Salpêtrière, 47-83

boulevard de l'hôpital, 75013

Paris, France;

maximel.bertoux@gmail.com

Received 16 November 2011 Revised 30 December 2011 Accepted 8 January 2012 Published Online First 29 January 2012

\section{ABSTRACT}

Background Behavioural variant of frontotemporal dementia (bvFTD) is a neurodegenerative disease that is clinically characterised by progressive behavioural changes and social interpersonal dysfunctions. Its diagnosis remains a clinical challenge, and depression is one of the main causes of misdiagnoses due to the prevalence of apathy in bvFTD.

Objective To evaluate the sensitivity and specificity of the Social Cognition and Emotional Assessment (SEA) and the mini-SEA for differentiating bvFTD from major depressive disorder (MDD).

Methods Scores for the SEA and mini-SEA for 37 patients with bvFTD (divided into subgroups of 17 with early bvFTD and 20 with moderate bvFTD according to the normal range of the Mattis Dementia Rating Scale), 19 MDD patients and 30 control subjects were compared to define the discrimination power of these tools compared with other standard neuropsychological tests.

Results SEA and mini-SEA scores were significantly lower for both the early and moderate bvFTD groups compared with control subjects and the MDD group, and very few scores overlapped between patients in the bvFTD subgroups and patients in the MDD and control subgroups. SEA and mini-SEA scores distinguished early bvFTD from MDD with sensitivity and specificity rates above $94 \%$

Conclusion Unlike standard executive neuropsychological tests, SEA and the mini-SEA can differentiate MDD from bvFTD in the early stages of the disease. The mini-SEA is an easy tool that can be utilised in neurological or psychiatric departments.

The behavioural variant of frontotemporal lobe degeneration (bvFTD) is a clinical syndrome involving focal atrophy that occurs predominantly in the frontal and temporal lobes and is associated with heterogeneous underlying pathologies. ${ }^{1}{ }^{2}$ In the absence of definitive biomarkers, the diagnosis is currently based on clinical criteria, which were recently revised. ${ }^{34}$ Symptoms are characterised by behavioural disinhibition, impaired social interaction, apathy or inertia, loss of empathy or sympathy, ${ }^{5}$ stereotyped or compulsive behaviour, and hyperorality or dietary changes; these factors are usually assessed using informant based interviews such as the Frontal Behavioural Inventory. ${ }^{6}$ In addition, neuropsychological assessments can highlight executive impairments, and some functions can be relatively spared or even normal, such as episodic memory, language, visuospatial functions and praxis. ${ }^{1}$ Conventional brain imaging tools are sometimes not sensitive enough for diagnostic validation in the early stage of the disease. ${ }^{7}$ The symptoms are quite insidious and can sometimes mimic psychiatric disorders. Thus diagnosing bvFTD remains a clinical challenge; furthermore, bvFTD patients may be misdiagnosed with psychiatric related disorders, such as depression, or they may be underdiagnosed or even be considered 'healthy'. 8

To improve diagnostic accuracy, tools such as tests of theory of mind have been proposed to assess social and emotional cognition, especially when classic executive tests show few abnormalities or normal performances. ${ }^{9-14}$ Although these tests have demonstrated effectiveness in distinguishing bvFTD patients from controls, their ability to discriminate bvFTD from depressive patients are not well known. As depression is one of the main misdiagnoses of bvFTD, ${ }^{8} 15^{17}$ it is essential to develop clinical tools that are be able to differentiate bvFTD from major depressive disorder (MDD).

In the present study, we studied the ability of the Social Cognition and Emotional Assessment $(\mathrm{SEA})^{14}$ and its shorter version, the mini-SEA, to differentiate MDD from bvFTD at both early and moderate stages.

\section{METHODS}

\section{Subjects}

Thirty-seven patients in the early or moderate stages of bvFTD, 19 patients with MDD and 30 controls were recruited for the study.

All bvFTD patients were evaluated at the Memory and Alzheimer's Institute at the PitiéSalpêtrière Hospital, Paris, France. Each patient's final diagnosis was established by FTD experts after multidisciplinary clinical meetings with neuropsychologists and neurologists. bvFTD patients were enrolled if they fulfilled the Lund and Manchester criteria for diagnosing bvFTD. ${ }^{3} 18$ All patients presented prominent changes in personality and social behaviour that were validated by their caregivers. All patients also underwent a standard neuropsychological examination, including the Mini-Mental State Examination (MMSE) and the Mattis Dementia Rating Scale (MDRS) ${ }^{19}$ for general cognitive functioning, the Frontal Assessment Battery (FAB) ${ }^{20}$ a verbal fluency test (semantic with animals and phonemic with the letter $M$ in $1 \mathrm{~min}$ ), 
the modified Wisconsin Card Sorting Task (WCST) for executive functions, the Free And Cued Selective Reminding Test ${ }^{21}$ for verbal episodic memory, a praxis evaluation and a word denomination task for language evaluation. Structural MRI and single photon emission computed tomography (SPECT) imaging examinations were performed for all patients; frontotemporal atrophy was identified on MRI and/or frontal hypoperfusion was detected in the SPECT images. Patients that presented with any of the following symptoms were not included in the study: (1) language deficit suggesting progressive non-fluent aphasia or semantic dementia, (2) a systemic illness that could interfere with cognitive functioning, (3) vascular lesions validated using MRI or neurological history suggesting vascular dementia or (4) a motor neuron disease. To improve diagnostic accuracy, all patients had at least one 18 month follow-up in the memory clinic of the Pitié-Salpêtrière Hospital to validate the diagnosis according to their clinical evolution.

We defined two subgroups of bvFTD patients according to their cognitive performance on the MDRS. This test is sensitive to frontal dysfunctions and is considered very useful for the cognitive assessment of bvFTD patients and for tracking disease progression. $^{22} 23$ The early bvFTD subgroup $(n=17)$ was defined as those receiving an MDRS score in the normal range (from 131 to 141, depending on age and educational level). The moderate bvFTD subgroup $(n=20)$ was defined as those receiving an MDRS score below the normal range (from 92 to 131, depending on age and educational level). We used French normative data for the MDRS

Nineteen patients with MDD were assessed at the Adult Psychiatry Departments of the Fernand-Widal-Lariboisière Hospital and the Pitié-Salpêtrière Hospital. Diagnoses were made according to the following criteria: (a) fulfilling the DSMIV criteria for MDD and (b) obtaining a Montgomery and Asberg Depression Rating Scale (MADRS) score $\geq 30 .{ }^{24}$ All patients were assessed by experienced psychiatrists. Exclusion criteria were: (1) a history of substance abuse, (2) a history of neurological disorders, (3) systemic illnesses that could interfere with cognitive functioning and (4) a history or evidence of psychotic symptoms or concomitant psychiatric disorders. All depressed patients underwent a reduced cognitive assessment that included the MMSE, FAB, a verbal morphological fluency evaluation and the modified WCST. Twenty-one patients were originally included. Two patients were eliminated from the study after a diagnostic change when a manic episode occurred post-evaluation. Seventeen patients were treated with selective serotonin reuptake inhibitors or serotonin-norepinephrine reuptake inhibitors, seven with tetracyclic antidepressants and one with agomelatine. In addition, 11 patients took typical neuroleptic drugs (phenothiazine or cyamemazine).

Thirty healthy control subjects, matched for age and education level, were included according to the following criteria: (1) an MMSE score $\geq 27 / 30$ and a FAB score $\geq 16 / 18$, (2) no history of neurological or psychiatric disorders and (3) no memory complaint or cognitive impairment.

For patients, all clinical data were obtained during the routine clinical work-up in the neurology and psychiatric departments and were extracted solely for the purpose of this study. Thus, according to French legislation, explicit informed consent was waived. However, the regulation concerning electronic filing was followed, and both patients and their relatives were informed that individual data may be used in retrospective clinical research studies. For healthy control subjects, the study was approved by the ethics committee for the protection of persons of the Pitié-Salpêtrière Hospital. All controls received oral and written information, and we obtained written informed consent before their participation.

\section{Social Cognition and Emotional Assessment}

All subjects underwent the same procedure.

The SEA consists of five subtests and provides six weighted composite scores: (1) a facial emotion recognition test (from Ekman pictures; scored from 0 to 15 ) in which patients must identify which emotion is being expressed, (2) a shortened version of the Faux Pas recognition test ${ }^{25}$ (scored from 0 to 15) that evaluates theory of mind, (3) a behavioural control test (scored from 0 to 5 ) in which patients must learn to apply a strategy of choice and to modify their choice based on monetary reward, (4) a reversal learning and extinction test (adapted from Rolls ${ }^{26}$ and scored from 0 to 5 ) in which patients must reverse a pattern of reinforced choice after contingencies are unexpectedly reversed and (5) an apathy scale from Starkstein ${ }^{27}$ (scored from 0 to 15). A general composite score was then calculated. The full details and explanations of the test designs, instructions and scoring methods are available in a previously published study. $^{14}$

We defined a mini-SEA comprising the sum of the facial emotion recognition and the Faux Pas test scores, which were validated in a previous study, to be able to accurately discriminate bvFTD from controls or patients with Alzheimer's disease (AD) ${ }^{14}$ The estimated test completion time for the mini-SEA was $30 \mathrm{~min}$. We chose not to include the apathy subscore as part of the mini-SEA because apathy is common to both MDD and bvFTD. ${ }^{8} 1518$ The behavioural control and reversal learning and extinction tests were not included in the mini-SEA because these tasks had a lower discriminating power between bvFTD and controls or $\mathrm{AD}$ patients. ${ }^{14}$

\section{Statistical analysis}

The normality of the demographic and neuropsychological data and of the SEA performances for all four groups was assessed using the Shapiro-Wilk test. Because the data were not normally distributed, we used a non-parametric statistical Kruskal-Wallis $\mathrm{H}$ test to compare the four groups, followed by the Mann-Whitney $U$ test to compare groups two by two. We used a modified formula of the Cohen $d$ test to evaluate the standardised difference between our means. For the correlation study, we used Spearman's rank correlation coefficient and applied a Bonferroni correction for multiple comparisons.

Receiver operating characteristics (ROC) curve analyses were used to evaluate the discriminating power of the SEA and miniSEA compared with the MMSE, FAB, verbal fluency test and number of perseverative errors on the WCST. The area under the curve (AUC) was used to measure the overall performance of each ROC curve (with 95\% CI). Finally, optimal cut-off points for the SEA were determined by selecting the point on the ROC curve where both sensitivity and specificity were maximised.

Statistical analyses were performed using STATISTICA 6 (http://www.statsoft.com) and MedCalc (http://www.medcalc. org) software.

\section{RESULTS}

Demographic data and neuropsychological performances of control subjects, MDD patients, and early and moderate bvFTD patients

All four groups were similar in age and educational level. MMSE and FAB scores were significantly higher in the control group compared with the MDD, early bvFTD and moderate bvFTD groups. The early bvFTD patients had significantly higher scores 
for all of the neuropsychological scores than the moderate bvFTD patients. We found no differences between the MDD and early bvFTD groups for any of the neuropsychological tests whereas the MDD group had significantly higher scores than the moderate bvFTD group for the FAB, WSCT and verbal fluency tests (see table 1).

\section{Comparison of SEA performances among groups}

SEA and mini-SEA scores and SEA subscores were significantly lower in both the early and moderate bvFTD groups compared with controls and the MDD group, except for the reversal learning and behavioural control tests. We found no significant differences in any of the SEA or mini-SEA scores between the early and moderate bvFTD groups. The results of the SEA composite score, reversal learning test and apathy scale were significantly lower in the MDD group compared with controls whereas no differences were found for the mini-SEA, emotion identification, theory of mind test or behavioural control test (table 2, figure 1).

Figure 1A shows scatterplots of the SEA composite scores across groups. Controls and MDD patients had the same score distribution, which was clearly distinct from the distribution observed for both early and moderate bvFTD patients. Figure 2 show scatterplots of the SEA and mini-SEA scores across the MDD and early bvFTD groups, which demonstrate very little overlap between the groups.

\section{Determining sensitivity, specificity and the optimal SEA cut-off scores for bvFTD diagnosis}

The ROC curve showed that the best tests for discriminating MDD from bvFTD patients were the SEA and mini-SEA. The SEA and mini-SEA had cut-off scores of 37.1 and 22.05 , respectively, that yielded the highest sensitivities $(91.9 \%$ and $89.2 \%$, respectively) and specificities (89.5\% and $100 \%$, respectively). The AUC was 0.97 for the SEA and 0.98 for the mini-SEA, which indicated that these tools have strong discriminating powers. The AUC for perseverative errors was 0.56 for the WCST, 0.66 for the MMSE, 0.75 for the FAB and 0.71 for verbal fluency (figures 1 and 2).

The sensitivity and specificity for differentiating early bvFTD from MDD were $94.1 \%$ and $89.5 \%$, respectively, for the SEA, and $94.1 \%$ and $100 \%$, respectively, for the mini-SEA, with cut-offs at
35.28 and 22.05, respectively. These values were similar to those for differentiating $\mathrm{MDD}$ patients from moderate bvFTD patients (sensitivity and specificity of $90 \%$ and $89.5 \%$ for the SEA and $85 \%$ and $100 \%$ for the mini-SEA, respectively). The AUC for perseverative errors was 0.55 for the WCST with $17.6 \%$ sensitivity and $100 \%$ specificity at a threshold of 11 . The AUC for the MMSE was 0.56 with $76.5 \%$ sensitivity and $36.8 \%$ specificity at a threshold of 25 . The AUC for the FAB was 0.57 with $64.7 \%$ sensitivity and $52.6 \%$ specificity at a threshold of 16 . The AUC for the verbal fluency test was 0.61 with $78.6 \%$ sensitivity and $50 \%$ specificity at a threshold of 10 .

The SEA and mini-SEA had similar diagnostic accuracy powers for distinguishing MDD from early bvFTD ( $d=3.86$ for the SEA and $d=3.26$ for the mini-SEA) and from moderate bvFTD ( $d=3.81$ and $d=2.68)$. The discrimination abilities of the SEA and mini-SEA were superior to the perseverative errors scores of the WCST $(\mathrm{d}=-0.32$ between MDD and early bvFTD; $\mathrm{d}=-0.64$ between MDD and moderate bvFTD), MMSE ( $d=0.17$ and $d=1.12), F A B(d=0.27$ and $d=1.28)$ and the verbal fluency test $(d=0.43$ and $d=0.72)$.

\section{Correlations between SEA and cognitive performances}

No correlations were observed between the SEA or mini-SEA subtests and the classic cognitive tests or MADRS, except for the performances of the $\mathrm{FAB}$ and the reversal learning test, which were significantly correlated $(r=0.79 ; \mathrm{p}<0.0001)$ in $\mathrm{MDD}$ patients.

\section{DISCUSSION}

We investigated the ability of the SEA to differentiate bvFTD from major depression. The SEA was used to assess social cognition and emotional processing dysfunctions that are caused by prefrontal lesions. Previous studies have demonstrated a high sensitivity and specificity of the test for differentiating bvFTD from controls or from patients with $\mathrm{AD} .{ }^{14}$ These findings are in accordance to those of other studies that have employed tests assessing executive functions and social cognition. ${ }^{12}$ However, an unresolved challenge was to understand whether these tests could discriminate bvFTD from depression. Here we show that the SEA and the mini-SEA differentiate both conditions with very high sensitivity and specificity.

Table 1 Characteristics and neuropsychological data of control subjects and patients

\begin{tabular}{|c|c|c|c|c|}
\hline Characteristics & Control group $(n=30)$ & MDD group $(n=19)$ & Early bvFTD $(n=17)$ & Moderate bvFTD $(n=20)$ \\
\hline Sex (M/F) & $13 / 17$ & $11 / 8$ & $11 / 6$ & $12 / 8$ \\
\hline Age (years) & $66.2 \pm 9.9(42-82)$ & $63.3 \pm 8.4(51-82)$ & $63.1 \pm 9.1(53-83)$ & $66.7 \pm 8.3(51-73)$ \\
\hline Education level & $10.7 \pm 3.7(5-16)$ & $10.5 \pm 4.7(2-17)$ & $10.8 \pm 3.9(3-15)$ & $10.4 \pm 4.2(5-17)$ \\
\hline \multicolumn{5}{|l|}{ Tests (maximal score) } \\
\hline MMSE (30) & $29 \pm 0.9(27-30)^{*} \dagger \neq$ & $26.7 \pm 2.2(23-30)^{*} \S$ & $27.1 \pm 2.3(21-30)+9$ & $23.3 \pm 3.9(15-28) \neq \S 9$ \\
\hline MDRS (144) & NA & NA & $135 \pm 3.2(131-141) 9$ & $119.2 \pm 12(92-131) \Phi$ \\
\hline FAB (18) & $17.1 \pm 1(16-18)^{*} \dagger \neq$ & $15.9 \pm 1.8(13-18)^{*} \S$ & $15.5 \pm 1.8(13-18) \dagger 9$ & $12.4 \pm 3.5(2-18) \neq \S 9$ \\
\hline WCST category (6) & NA & $4.7 \pm 1.4(2-6) \S$ & $5 \pm 1.8(2-6) 9$ & $3.1 \pm 1.9(0-6) \S \uparrow$ \\
\hline WCST perseveration errors & NA & $3.1 \pm 3.6(0-11)$ & $4.7 \pm 6.3(0-14)$ & $5.8 \pm 4.9(0-14)$ \\
\hline WCST attentional errors & NA & $2.2 \pm 1.7(0-6)^{* *}$ & $0.9 \pm 1.5(0-4)^{* *}$ & $1.8 \pm 1.7(0-5)$ \\
\hline Morphological fluency & NA & $10.8 \pm 4.5(2-20) \S$ & $9.1 \pm 3.6(4-15) \Phi$ & $3.9 \pm 6.4(1-30) \S$ ฯ \\
\hline \multicolumn{5}{|c|}{$\begin{array}{l}\text { Results are expressed as mean } \pm \text { SD (range). } \\
\text { *Significant difference between control subjects and MDD. MMSE: } p<0.0001 ; F A B: p<0.05 \text {. } \\
\text { †Significant difference between control subjects and early bvFTD. MMSE: } p<0.001 ; F A B: p<0.001 \text {. } \\
\text { †Significant difference between control subjects and moderate bvFTD. MMSE: } p<0.00001 ; \text { FAB: } p<0.00001 \text {. } \\
\text { §Significant difference between MDD and moderate bvFTD. MMSE: } p<0.0001 ; F A B: p<0.0001 ; \text { WCST category: } p=0.01 ; \text { fluency: } p<0.005 \text {. } \\
\text { } \text { Significant difference between early bvFTD and moderate bvFTD. MMSE: } p<0.001 ; F A B: p<0.005 ; \text { WCST category: } p<0.005 ; \text { fluency: } p=0.005 ; \text { MDRS: } p<0.00001 \text {. } \\
\text { ** Significant difference between MDD and early bvFTD. Attention errors in WCST: } p=0.01 \text {. } \\
\text { bvFTD, behavioural version of frontotemporal dementia; FAB, Frontal Assessment Battery; MDD, major depressive disorder; MDRS, Mattis Dementia Rating Scale; MMSE, Mini-Mental State } \\
\text { Examination; WCST, Wisconsin Card Sorting Task. }\end{array}$} \\
\hline
\end{tabular}


Table 2 Social Cognition and Emotional Assessment performances

\begin{tabular}{|c|c|c|c|c|}
\hline Tests (maximal score) & Control group & MDD & Early bvFTD & Moderate bvFTD \\
\hline SEA composite (55) & $47.2 \pm 3.8(40.1-53.1)^{*} \dagger \neq$ & $42.6 \pm 4.8(33.5-51.4)^{*} \S$ ๆ & $29.2 \pm 5.7(15.7-38.8)+$ ฯ & $28.9 \pm 5.8(20.5-39.4) \neq \S$ \\
\hline Emotion identification (15) & $12.6 \pm 1.1(10.2-14.6) \dagger \neq$ & $12.4 \pm 0.7(11.6-13.7) \S 9$ & $9.7 \pm 1.8(6.8-12.9) \dagger$ ฯ & $8.4 \pm 2.4(3.9-11.1) \neq \S$ \\
\hline Faux Pas recognition test (15) & $13.2 \pm 1.5(9-15) \dagger \neq$ & $13.3 \pm 1.4(10.8-13.3) \S$ ๆ & $8.4 \pm 2.3(4.1-13.5) \dagger$ ศ & $9.6 \pm 2.2(5.6-14.3) \neq \S$ \\
\hline Behavioural control (5) & $3.2 \pm 1.2(0.5-5) \dagger \neq$ & $2.5 \pm 1.1(0.5-4) \S$ & $1.8 \pm 1.1(0-3.5) \dagger$ & $1.6 \pm 1.5(0-5) \neq \S$ \\
\hline Reversal learning (5) & $3.5 \pm 1.9(0-5)^{*} \dagger \neq$ & $2.6 \pm 1.9(0-5)^{*}$ & $1.5 \pm 1.7(0-4.5) \dagger$ & $1.6 \pm 1.5(0-4) \ddagger$ \\
\hline Apathy scale (15) & $15 \pm 0(15-15)^{*} \dagger \neq$ & $11.8 \pm 3.6(6.4-15)^{*} \S 9$ & $9.2 \pm 3.6(2.4-15) \dagger$ ฯ & $7.9 \pm 2.9(3.6-15) \neq \S$ \\
\hline Mini-SEA composite (30) & $25.8 \pm 1.8(21.6-29.6) \dagger \neq$ & $25.7 \pm 1.7(22.8-28.7) \S 9$ & $18.1 \pm 3(11.8-23.6) \dagger$ ฯ & $18 \pm 3.8(11.4-25.4) \ddagger \S$ \\
\hline
\end{tabular}

Results are expressed as mean \pm SD (range)

*Significant difference between control subjects and MDD. SEA: $p<0.001$; reversal learning test: $p<0.05$; apathy scale: $p<0.0001$

†Significant difference between control subjects and early bvFTD. SEA: $p<0.0001$; emotion recognition: $p<0.0001 ;$ Faux Pas test: $p<0.0001 ;$ apathy scale: $p<0.0001$.

¥Significant difference between control subjects and moderate bvFTD. SEA: $p<0.0001$; emotion recognition: $p<0.0001 ;$ Faux Pas test: $p<0.0001$; apathy scale: $p<0.0001$.

$\S$ Significant difference between MDD and moderate bvFTD. SEA: $p<0.0001$; emotion recognition: $p<0.0001$; Faux Pas test: $p<0.0001$; behavioural control test: $p<0.01$; reversal learning test: $p<0.05$; mini-SEA: $p<0.0001$.

-Significant difference between MDD and early bvFTD. SEA: $p<0.0001$; emotion recognition: $p<0.0001$; Faux Pas test: $p<0.0001 ;$ mini-SEA: $p<0.0001$.

bvFTD, behavioural version of frontotemporal dementia; MDD, major depressive disorder; SEA, Social Cognition and Emotional Assessment.

Several studies have demonstrated that early impairments in social and emotional cognition occur in bvFTD. ${ }^{9-14}$ Although bvFTD is often misdiagnosed as depression, ${ }^{8}$ no data on the discriminating power of this type of test for differentiating MDD from bvFTD are available.

Early bvFTD patients and MDD patients can exhibit similar behavioural changes, such as apathy and inertia; apathy is present in $60-90 \%$ of bvFTD cases. $^{28} 29$ In addition to apathy and inertia, MDD patients exhibit executive impairments, such as impairments in concept generation, ${ }^{30}$ inhibition, ${ }^{31}$ sustained attention, ${ }^{32}$ free memory recall, ${ }^{33}$ working memory ${ }^{34}$ and verbal fluency. ${ }^{35}$ These cognitive processes can also be impaired in bvFTD patients. Early bvFTD can therefore be mistaken for depression, especially when neuropsychological profiles are similar between the conditions. ${ }^{16} 17$ Moreover, brain imaging can fail to provide useful information for the differential diagnosis; MRIs may be normal in the early stages of bvFTD, whereas SPECT and fluorodeoxyglucose positron emission tomography can show frontal hypoperfusion/hypometabolism in MDD. ${ }^{36-38}$

The results of this study demonstrated that the SEA, and particularly the mini-SEA, can efficiently differentiate MDD from bvFTD, even at an early stage of the disease when
Figure 1 Scatterplots showing SEA and mini-SEA composite scores and scores across groups. (A) Scatterplots showing SEA composite scores across groups. (B) Scatterplots showing miniSEA composite scores across the MDD and early bvFTD groups. (C) Scatterplots showing SEA composite scores across the MDD and early bvFTD groups. bvFTD, behavioural variant of frontotemporal dementia; MDD, major depressive disorder; SEA, social cognition and emotional assessment.
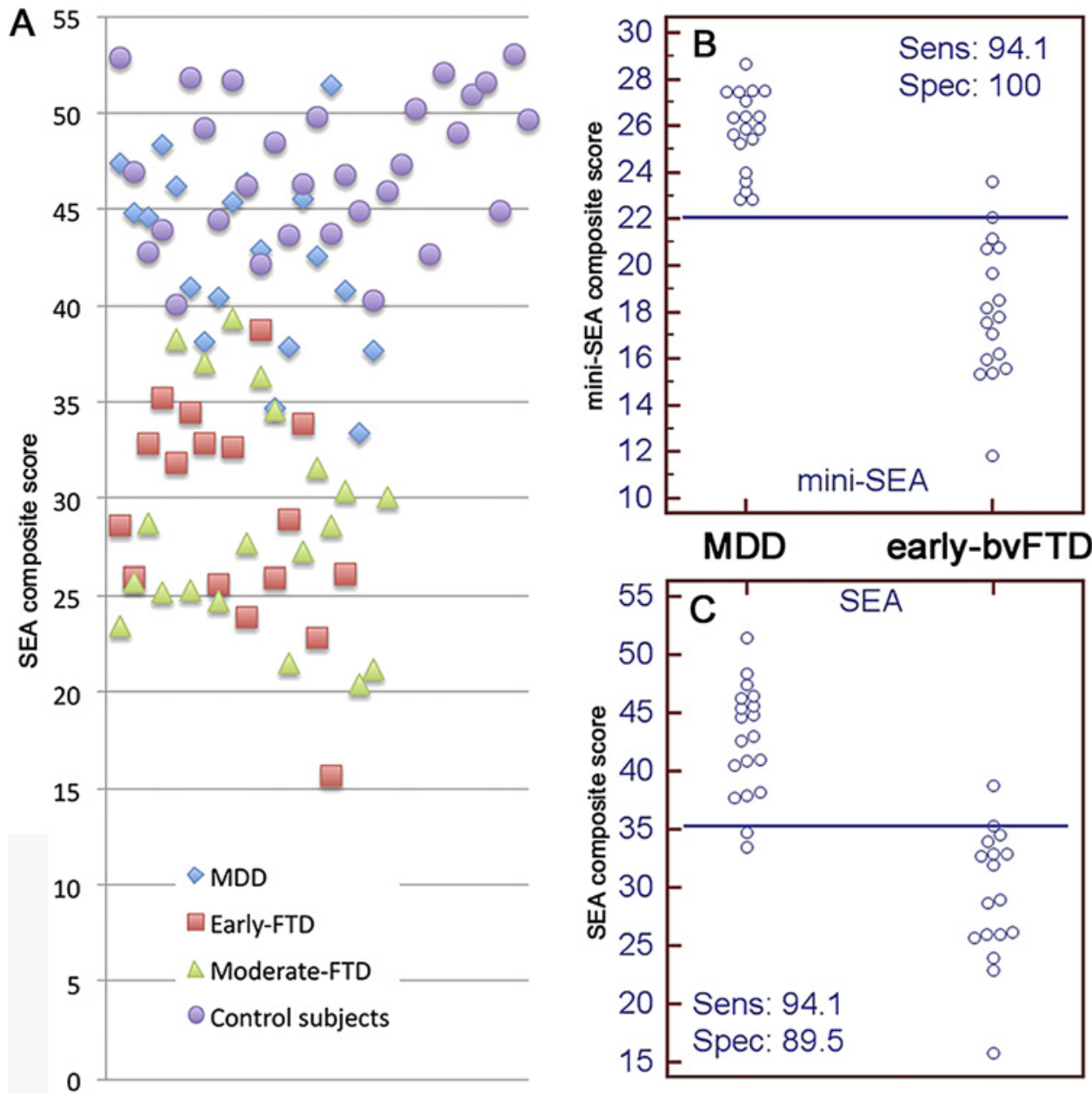


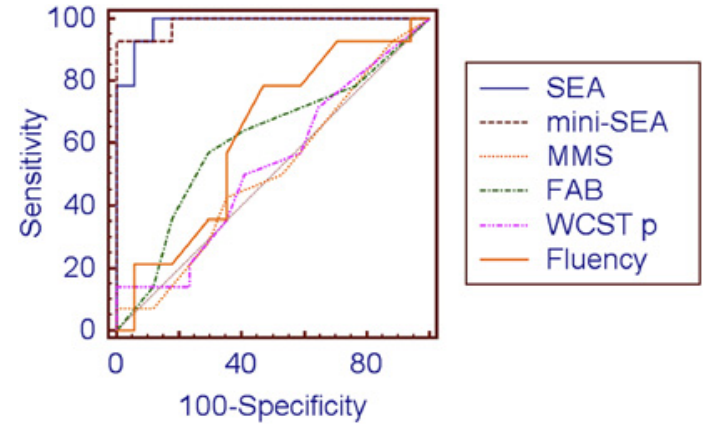

Figure 2 Receiver operating characteristic curves for the SEA and mini-SEA composite scores and executive or general neuropsychological tests for MDD patients compared with those for early bvFTD patients. A cut-off score of 35.28 for the SEA test differentiated the early bvFTD group from the MDD groups with $94.1 \%$ sensitivity and $89.5 \%$ specificity. bvFTD, behavioural variant of frontotemporal dementia; $F A B$, Frontal Assessment Battery; MDD, major depressive disorder; MMS, Mini-Mental State Examination; SEA, Social Cognition and Emotional Assessment; WCST, Wisconsin Card Sorting Task.

neuropsychological tests are still normal. By classifying bvFTD patients as being above or below the normal range in the MDRS, the SEA and mini-SEA differentiated MDD from early bvFTD patients, even though the patient groups did not exhibit any differences in the neuropsychological tests that assess executive functions. For the moderate bvFTD group, the best test for the differential diagnosis remained the SEA compared with classical executive tests. Yet it is noteworthy that the MDRS can remain normal for an extended time during the course of the disease and cannot be considered as a perfect reliable marker of disease severity.

The SEA was constructed by including tasks that are known to be impaired early in bvFTD patients and to be associated with damage to the neural network involving the orbitofrontal and medial prefrontal regions: theory of mind, ${ }^{9-14}$ reversal learning and behavioural control tests, ${ }^{9} 1428$ apathy evaluation ${ }^{14}{ }^{39}$ and facial emotion recognition assessment. ${ }^{14} 40$ We defined a miniSEA to develop a quick and easy clinical test that could be administered to detect subtle relevant changes that are caused by bvFTD but not by MDD. The mini-SEA takes approximately 30 min to administer. Both the SEA and mini-SEA were similarly effective for differentiating MDD from early bvFTD and can be easily administered in neurological or psychiatric departments. The diagnostic value of the mini-SEA for differentiating early bvFTD from MDD is strengthened by the observations: (1) that the mini-SEA performances were similar between controls and MDD patients, (2) that the SEA and mini-SEA scores did not correlate with severity of depression, which was assessed by the MADRS, (3) that only one patient diagnosed with early bvFTD had a mini-SEA score overlapping with scores of depressive patients, (4) that the SEA was not correlated with scores for tests of executive functions, suggesting that these tests assess distinct processes and (5) that the SEA performances were equally decreased in patients with early and moderate bvFTD, demonstrating the test's assessment value at the onset of the disease.

Simple and specific tests of emotional and social cognition, including tests of theory of mind, are lacking in their application to FTD. The mini-SEA is an easy and fast tool that can be utilised in neurological or psychiatric departments.

Although pathological data may help to establish the diagnosis of the different subgroups of bvFTD patients, no post mortem data were available in our cohort. Future autopsy studies are needed to validate the diagnoses and confirm the findings. However, to decrease the risk of false diagnoses, all FTD patients underwent a complete neurological evaluation, including brain imaging, and were followed for at least 18 months to validate their diagnosis by the evolution of clinical symptoms.

\section{CONCLUSION}

The development of care management strategies needs to improve the differential diagnosis between MDD and bvFTD. Overall, this study showed that the SEA and mini-SEA are useful tools for early cognitive assessments and are relevant for use in daily clinical practice. The SEA can detect specific features of early bvFTD when classic neuropsychological tests are still normal. Finally, the results of this study are in accordance with the growing number of studies that have demonstrated the relevance of early and specific impairments in social and emotional cognition in bvFTD, which may be included in future revisions of bvFTD diagnostic criteria.

Acknowledgements The authors are grateful to Romain Icick, Sophie Guillonet, Floriane Chauvin and Beatrice Garcin for help in the recruitment of patients. Thanks also go to Raphaël Gaillard, Severine Hatif, Magali Seassau and Serge Kinkingnehun for comments on an earlier version of this project.

Contributors MB: design, clinical and experimental data acquisition, analysis and interpretation, and manuscript writing. Statistical analyses were performed by MB. MD: clinical data acquisition and manuscript revision. LCS: manuscript revision and experimental data interpretation. AF: design, manuscript revision and experimental data interpretation. J-PL: manuscript revision, and experimental and clinical data interpretation. PF: manuscript revision and clinical data interpretation. BD: manuscript revision and clinical data interpretation. MS: manuscript writing, clinical and experimental data analysis and interpretation.

Funding MB was supported by the French Ministry of Defence and the National Centre for Scientific Research (CNRS) during his PhD.

Competing interests LCS received speaker honoraria from Lundbeck. J-PL received lecture honoraria from Servier, Sanofi, Pfizer-Wyeth and Pierre Fabre. PF received honoraria from Servier, Lundbeck, Eli Lilly and research grants from Servier. BD has consulted or served on advisory board for Bristol-Myers Squibb, Roche, Elan, Eli Lilly, Eisai and Janssen. His institution has received grants from Novartis and

Sanofi-Aventis. MS received speaker honoraria from EISAl, Pfizer, Lundbeck, Janssen and Novartis; she belongs to a scientific advisory board for EISAI Company and serves as an associate editor for La Lettre du Neurologue.

Patient consent For patients, all clinical data were obtained during routine clinical work-up in the neurology and psychiatric departments and were extracted solely for the purpose of this study. Thus, according to French legislation, explicit informed consent was waived. However, the regulation concerning electronic filing was followed, and both patients and their relatives were informed that individual data may be used in retrospective clinical research studies. For healthy control subjects, the study was approved by the ethics committee for the protection of persons of the Pitié-Salpêtrière Hospital. All controls received oral and written information and we obtained a signed informed consent form before their participation.

Ethics approval The study was approved by the ethics committee of Pitié-Salpêtrière Hospital, France.

Provenance and peer review Not commissioned; externally peer reviewed.

\section{REFERENCES}

1. Piguet $\mathbf{0}$, Hornberger M, Mioshi E, et al. Behavioural-variant frontotemporal dementia: diagnosis, clinical staging, and management. Lancet Neurol 2011:10:162-72

2. Mackenzie IR, Neumann M, Bigio EH, et al. Nomenclature and nosology for neuropathologic subtypes of frontotemporal lobar degeneration: an update. Acta Neuropathol 2010;119:1-4.

3. Neary D, Snowden JS, Gustafson L, et al. Frontotemporal lobar degeneration: a consensus on clinical diagnostic criteria. Neurology 1998;51:1546-54.

4. Rascovsky K, Hodges JR, Knopman D, et al. Sensitivity of revised diagnostic criteria for the behavioural variant of frontotemporal dementia. Brain 2011;134:2456-77.

5. Rankin KP, Kramer JH, Miller BL. Patterns of cognitive and emotional empathy in frontotemporal lobar degeneration. Cogn Behav Neurol 2005;18:28-36. 
6. Kertesz A, Nadkarni N, Davidson W, et al. The Frontal Behavioral Inventory in the differential diagnosis of frontotemporal dementia. J Int Neuropsychol Soc 2000;6:460-8.

7. Mendez MF, Shapira JS, McMurtray A, et al. Accuracy of the clinical evaluation for frontotemporal dementia. Arch Neurol 2007;64:830-5.

8. Woolley JD, Khan BK, Murthy NK, et al. The diagnostic challenge of psychiatric symptoms in neurodegenerative disease: rates of and risk factors for prior psychiatric diagnosis in patients with early neurodegenerative disease. J Clin Psychiatry 2011:72:126-33.

9. Rahman S, Sahakian BJ, Hodges JR, et al. Specific cognitive deficits in early frontal variant frontotemporal dementia. Brain 1999;122:1469-93.

10. Lough S, Kipps CM, Treise $C$, et al. Social reasoning, emotion and empathy in frontotemporal dementia. Neuropsychologia 2006;44:950-8.

11. Torralva T, Kipps CM, Hodges JR, et al. The relationship between affective decisionmaking and theory of mind in the frontal variant of fronto-temporal dementia. Neuropsychologia 2007; 45:342-9.

12. Torralva T, Roca M, Gleichgerrcht $\mathrm{E}$, et al. A neuropsychological battery to detect specific executive and social cognitive impairments in early frontotemporal dementia. Brain 2009;132:1299-309.

13. Adenzato M, Cavallo M, Enrici I. Theory of mind ability in the behavioural variant of frontotemporal dementia: an analysis of the neural, cognitive, and social levels. Neuropsychologia 2010;48:2-12.

14. Funkiewiez A, Bertoux M, de Souza LC, et al. The SEA (social cognition and emotional assessment): a clinical neuropsychological tool for early diagnosis of frontal variant of frontotemporal lobar degeneration. Neuropsychology 2012;26:81-90.

15. Miller BL, Cummings JL, Villanueva-Meyer J, et al. Frontal lobe degeneration: clinical, neuropsychological, and SPECT characteristics. Neurology 1991;41:1374-82

16. Braaten AJ, Parsons TD, McCue R, et al. Neurocognitive differential diagnosis of dementing diseases: Alzheimer's dementia, vascular dementia, frontotemporal dementia, and major depressive disorder. Int J Neurosci 2006;116:1271-93.

17. Elderkin-Thompson V, Boone KB, Hwang S, et al. Neurocognitive profiles in elderly patients with frontotemporal degeneration or major depressive disorder. J Int Neuropsychol Soc 2004;10:753-71.

18. McKhann GM, Albert MS, Grossman M, et al; Work Group on Frontotemporal Dementia and Pick's Disease. Clinical and pathological diagnosis of frontotemporal dementia: report of the Work Group on Frontotemporal Dementia and Pick's Disease. Arch Neurol 2001:58:1803-9.

19. Mattis S. Mental status examination for organic mental syndrome in the elderly patients. In: Bellak L, Karasu T, eds. Geriatrics psychiatry: a handbook for psychiatrists and primaty care physicians. New York: Grune \& Stratton, 1976:77-121.

20. Dubois B, Slachevsky A, Litvan I, et al. The FAB: a frontal assessment battery at bedside. Neurology 2000;55:1621-6.

21. Van der Linden M. L'évaluation des troubles de la mémoire - Présentation de qutre tests de mémoire épisodique (avec leur étalonnage). Paris: Solal, 2006.

22. Pasquier F. Early diagnosis of dementia: neuropsychology. J Neurol 1999;246:6-15.
23. Rascovsky K, Salmon DP, Hansen LA, et al. Distinct cognitive profiles and rates of decline on the Mattis Dementia Rating Scale in autopsy-confirmed frontotemporal dementia and Alzheimer's disease. J Int Neuropsychol Soc 2008;14:373-83.

24. Muller MJ, Himmerich $\mathrm{H}$, Kienzle B, et al. Differentiating moderate and severe depression using the Montgomery-Asberg depression rating scale (MADRS). J Affect Disord 2003:77:255-60.

25. Stone VE, Baron-Cohen S, Knight RT. Frontal lobe contributions to theory of mind. $J$ Cogn Neurosci 1998;10:640-56.

26. Hornak J, O’Doherty J, Bramham J, et al. Reward-related reversal learning after surgical excisions in orbito-frontal or dorsolateral prefrontal cortex in humans. J Cogn Neurosci 2004;16:463-78.

27. Starkstein SE, Mayberg HS, Preziosi TJ, et al. Reliability, validity, and clinica correlates of apathy in Parkinson's disease. J Neuropsychiatry Clin Neurosci 1992; 4:134-9.

28. Le Ber I, Guedj E, Gabelle A, et al. Demographic, neurological and behavioural characteristics and brain perfusion SPECT in frontal variant of frontotemporal dementia. Brain 2006:129:3051-65.

29. Chow TW, Binns MA, Cummings JL, et al. Apathy symptom profile and behaviora associations in frontotemporal dementia vs dementia of Alzheimer type. Arch Neurol 2009:66:888-93

30. Fossati $\mathbf{P}$, Ergis AM, Allilaire JF. Problem-solving abilities in unipolar depressed patients: comparison of performance on the modified version of the Wisconsin and the California sorting tests. Psychiatry Res 2001;104:145-56.

31. Elderkin-Thompson V, Mintz J, Haroon E, et al. Executive dysfunction and memory in older patients with major and minor depression. Arch Clin Neuropsychol 2007:22:261-70.

32. Weiland-Fiedler P, Erickson K, Waldeck T, et al. Evidence for continuing neuropsychological impairments in depression. J Affect Disord 2004:82:253-8.

33. Fossati P, Harvey PO, Le Bastard G, et al. Verbal memory performance of patients with a first depressive episode and patients with unipolar and bipolar recurrent depression. J Psychiatr Res 2004;38:137-44.

34. Harvey PO, Le Bastard G, Pochon JB, et al. Executive functions and updating of the contents of working memory in unipolar depression. J Psychiatr Res 2004:38:567-76.

35. Fossati P, Guillaume le B, Ergis AM, et al. Qualitative analysis of verbal fluency in depression. Psychiatry Res 2003;117:17-24.

36. Gregory CA, Serra-Mestres J, Hodges JR. Early diagnosis of the frontal variant of frontotemporal dementia: how sensitive are standard neuroimaging and neuropsychologic tests? Neuropsychiatry Neuropsychol Behav Neurol 1999;12:128-35

37. Perry RJ, Graham A, Williams G, et al. Patterns of frontal lobe atrophy in frontotemporal dementia: a volumetric MRI study. Dement Geriatr Cogn Disord 2006;22:278-87.

38. Monkul ES, Silva LA, Narayana S, et al. Abnormal resting state corticolimbic blood flow in depressed unmedicated patients with major depression: a (15) 0-H(2) 0 PET study. Hum Brain Mapp 2012;33:272-9.

39. Zamboni G, Huey ED, Krueger F, et al. Apathy and disinhibition in frontotemporal dementia: insights into their neural correlates. Neurology 2008;71:736-42.

40. Heberlein AS, Padon AA, Gillihan SJ, et al. Ventromedial frontal lobe plays a critica role in facial emotion recognition. J Cogn Neurosci 2008;20:721-33. 